\title{
Validez interna
}

\section{Internal validity}

\author{
Rocío B. Mayorga-Ponce ${ }^{a}$, Dania Z. Plata-Balderas ${ }^{b}$ Abigail Martínez-Alamilla $^{c}$, Daniela \\ Salazar-Valdez ${ }^{d}$
}

\begin{abstract}
:
Internal validity is the degree of confidence that the results of the experiment are properly interpreted and valid, this in turn relates to the quality of the experiment and is achieved when there is control, when the groups differ from each other only in exposure to the independent variable, when the measurements of the dependent variable are reliable and valid, and when the analysis is appropriate for the type of data we are handling.
\end{abstract}

Keywords:

Internal, validity, confidence, experiment, measurements

\section{Resumen:}

La validez interna es el grado de confianza que se tiene de que los resultados del experimento se interpreten adecuadamente y sean válidos, este a su vez se relaciona con la calidad del experimento y se logran cuando hay control, cuando los grupos difieren entre sí solamente en la exposición a la variable independiente, cuando las mediciones de la variable dependiente son confiables y válidas y cuando el análisis es el adecuado para el tipo de datos que estamos manejando.

Palabras Clave:

Interna, validez, confianza, experimento, mediciones

\section{Introducción}

El ontrol en un experimento logra la validez interna y esto se puede alcanzar mediante varios grupos de comparacion, en los cuales deben existir dos como mínimo, además de la equivalencia de los grupos en todo, excepto en la manipulación de la o las variables independientes. ${ }^{1}$
El necesitar de mínimo dos grupos es para poder comparar los resultados, ya que, si se experimentara con tan solo un grupo, sería imposible saber si hubo otro factor de influencia ajeno a la variable independiente que se manipuló, además de que ambos grupos deberán cumplir con las mismas características. ${ }^{2}$

Validez interna.

\footnotetext{
Rocío Belem Mayorga Ponce, Universidad Autónoma del Estado de Hidalgo, https://orcid.org/000-0003-3544-7171, Email: rmponce@uaeh.edu.mx

b Dania Zereth Plata Balderas. Universidad Autónoma del Estado de Hidalgo, https://orcid.org/0000-000-2793-6301X, Email: pl284568@uaeh.edu.mx

Abigail Martínez Alamilla Universidad Autónoma del Estado de Hidalgo, https://orcid.org/0000-0002-4712-6351, Email: abigailmartinez5809@gmail.com

d Daniela Salazar Valdez Universidad Autónoma del Estado de Hidalgo, https://orcid.org/0000-0002-5517-0811, Email danielasalazar457@gmail.com
} 
La validez interna es una medida crucial en los estudios cuantitativos, donde asegura que el diseño del experimento de un investigador sigue de cerca el principio de causa y efecto. La forma más sencilla de describir la validez interna es la confianza que podemos depositar en la relación causa-efecto en un estudio. ${ }^{3}$

La validez interna va a depender de los procedimientos de un estudio y rigurosidad con que se realice. Su objetivo principal es el considerar la seguridad de los resultados del estudio.

Cuanto menor sea la posibilidad de "confusión" en un estudio, mayor será la validez interna y más confianza tendremos en los hallazgos. La confusión se refiere a una situación en la que entran en juego otros factores que confunden el resultado de un estudio, por ejemplo, un estudio podría hacernos inseguros de si podemos confiar en que hemos identificado el escenario de "causa y efecto" anterior. ${ }^{4}$

La invalidación interna también puede existir si las variables independientes afectan a las dependientes, por ejemplo:

1.- Historia: cuando hay acontecimientos externos que ocurren simultaneamente con éste y que pueden alterar 0 influir.

2.- Factores de confusión: se refiere a una situación en la que existe la posibilidad de que algunos otros factores entren en juego en el estudio de investigación y, por lo tanto, produzcan confusión en cuanto a qué factores de hecho están dando los resultados, estos factores de confusión también llamados variables extrañas son los que amenazan la validez interna de la investigación.

3.- Selección: cuando los grupos de estudio son diferentes o no equiparables. Ej. Raza, sexo, etc.

4.- Maduración: son los cambios producidos por evolución natural. Tiene relevancia en salud y confunde el efecto del cambio de la variable con el de la causa.

5.- Efectos relativos del pre-test: es la influencia que produce el pre-test.

6.- Difusión: un tratamiento puede extenderse del tratamiento al grupo de control, ya sea a través de los miembros del grupo interactuando, hablando 0 simplemente observándose entre sí, esto afecta la validez interna de la investigación.

7.- Mortalidad: el que desaparezcan sujetos de los grupos de comparación.

8.- Instrumentación: uso de instrumentos no fiables ni validos.

9.- Regresión estadística: los sujetos seleccionados representan situaciones o puntuaciones en alguna variable. Cuando se usan sujetos externos.

10.- Sesgo del experimentador: esto se refiere al mismo experimentador actuando y comportándose de manera diferente hacia los diferentes grupos de estudio, lo que en última instancia tendría un impacto en los resultados del estudio..$^{5,6}$

Para mejorar la validez interna es necesario tomar en cuenta:

1.- Aleatorización y selección: una de las prácticas más conocidas para aumentar la validez interna es seleccionar primero a los participantes al azar. Después de la selección, asignar nuevamente al azar a los participantes de la investigación al tratamiento y a los grupos de control. 2.- Protocolo de estudio: si los investigadores siguen el conjunto específico de procedimientos para la administración del tratamiento o la intervención, pueden aumentar la validez interna de un estudio determinado.

3.- Manipulación experimental: es probable que la validez interna mejore si un investigador manipula la variable independiente en lugar de simplemente observar la relación sin introducir la intervención o el tratamiento.

4.- Cegamiento: se refiere a ocultar el conocimiento sobre la intervención o el tratamiento que se está introduciendo a los participantes y, a veces, a los propios investigadores para que no sesgue sus comportamientos y percepciones, afectando así los resultados del estudio. ${ }^{(6)}$

\section{Conclusión.}

El principal objetivo de la validez interna es el considerar la seguridad de los resultados del estudio, además de ser utilizada para validar un estudio que será aplicado a dos o más grupos, pero en diferentes momentos. Dichos grupos deben cumplir con las mismas características y el contar con dos grupos como mínimo es para poder ver las diferencias entre uno y otro y ver de qué modo es que influyeron las variables. Para llevar a cabo la validez interna es necesario tomar en cuenta algunos aspectos importantes, ya que de no hacerlo, no podrían ser validos los resultados.

\section{Referencias}

[1] Sampieri. RH. ¿Cómo se logran el control y la validez interna?. In Martínez. MIR, editor. Metodología de la investigación.. CDMX: McGraw Hill.; 2014. p. 137-138.

[2] Rodríguez. D. Lifeder.com.2019 Recuperado el 18 de octubre de 2020.Disponible en : https://www.lifeder.com/validez-interna/.

[3] Shuttleworth. M. Validez interna. 2020 . Recuperado el 18 de octubre de 2020. Disponible en: https://explorable.com/internalvalidity\#: :text=\%20Internal $\% 20$ Validity $\% 20$ -

$\% 20$ the $\% 20$ Final $\% 20$ Word $\% 20$,of $\% 20$ the $\% 20$ children $\% 20$ in $\% 20$ th e\%20computerless. \%20More\%20. 
Publicación semestral, Educación y Salud Boletín Científico Instituto de Ciencias de la Salud Universidad Autónoma del Estado de Hidalgo, Vol. 9, No. 18 (2021) 68-70

[4] Cunic. A. Comprensión de la validez interna y externa.2020. Recuperado el 18 de Octubre de 2020. Disponible en : https://www.verywellmind.com/internal-and-external-validity4584479 .

[5] Quezada. ME. Diseño de investigación, validez interna y validez externa. 2016.Recuperado el 18 de octubre de 2020. Disponible en: https://www.slideshare.net/Lizherireth/diseo-de-investigacinvalidez-interna-y-validez-externa.

[6] Naeem. S. Validez interna en la investigación. 2019 Recuperado el 18 de octubre de 2020. Disponible en http://researcharticles.com/index.php/internal-validity-research/. 